The present results confirm an earlier study ${ }^{11}$ which concluded that the atmosphere-ocean system contains critical levels of atmospheric $\mathrm{CO}_{2}$ beyond which irreversible changes of the Atlantic thermohaline circulation are likely. Such changes would have also important direct and indirect consequences for the distribution of antropogenic carbon: a strong reduction of ventilation decreases the transport of excess carbon and heat into the deep ocean. The latter leads to an enhanced warming of the upper layers of the ocean which, in turn, decreases the solubility of $\mathrm{CO}_{2}$ and results in a further increase of atmospheric $\mathrm{CO}_{2}$. This feedback mechanism has the tendency to further destabilize the thermohaline circulation.

Our finding, that these critical levels also depend on the rate of $\mathrm{CO}_{2}$ increase, is also relevant for decisions about the choice of a particular scenario of future greenhouse gas emissions. In the future, such decisions will not only need to consider the stabilization concentration or the economic cost of emission reduction to reach this concentration ${ }^{29}$, but also take into account critical limits on the rate of greenhouse gas increase of the atmosphere.

Received 21 April; accepted 30 June 1997

Houghton, J. T. et al. (eds) Climate Change 1995: The Science of Climate Change (Cambridge Univ. Press, 1996).

2. Oeschger, H. et al. in Climate Processes and Climate Sensitivity (eds Hansen, J. E. \& Takahashi, T.) 299306 (Geophys. Monogr. 29, Am. Geophys. Un., Washington DC, 1984).

Broecker, W. S., Peteet, D. \& Rind, D. Does the ocean-atmosphere system have more than one stable mode of operation? Nature 315, 21-25 (1985).

4. Broecker, W. S. \& Denton, G. H. The role of ocean-atmosphere reorganizations in glacial cycles. Geochim. Cosmochim. Acta 53, 2465-2501 (1989).

Bond, G. et al. Correlations between climate records from North Atlantic sediments and Greenland ice. Nature 365, 143-147 (1993).

6. Bryan, F. High-latitude salinity effects and interhemispheric thermohaline circulations. Nature $\mathbf{3 2 3}$ 301-304 (1986)

Manabe, S. \& Stouffer, R. J. Two stable equilibria of a coupled ocean-atmosphere model. J. Clim. 1, $841-866$ (1988).

8. Maier-Reimer, E. \& Mikolajewicz, U. Experiments with an OGCM on the cause of the Younger Dryas 113 (Tech. Rep. 39, Max-Planck-Inst. für Meteorol., Hamburg, 1989).

9. Stocker, T. F. \& Wright, D. G. Rapid transitions of the ocean's deep circulation induced by changes in surface water fluxes. Nature 351, 729-732 (1991).

10. Manabe, S., Stouffer, R. J., Spelman, M. J. \& Bryan, K. Transient responses of a coupled oceanatmosphere model to gradual changes of atmospheric $\mathrm{CO}_{2}$. Part I: Annual mean response. J. Clim. 4, 785-818 (1991).

11. Manabe, S. \& Stouffer, R. J. Century-scale effects of increased atmospheric $\mathrm{CO}_{2}$ on the oceanatmosphere system. Nature 364, 215-218 (1993).

12. Manabe, S. \& Stouffer, R. J. Multiple-century response of a coupled ocean-atmosphere model to an increase of atmospheric carbon dioxide. J. Clim. 7, 5-23 (1994).

13. Sarmiento, J. L. \& Le Queré, C. Oceanic carbon dioxide in a model of century-scale global warming. Science 274, 1346-1350 (1996).

14. Neftel, A., Oeschger, H., Staffelbach, T. \& Stauffer, B. $\mathrm{CO}_{2}$ record in the Byrd ice core 50,000-5,000 years B. Nature 331, 609-611 (1988)

15. Broecker, W. S. Unpleasant surprises in the greenhouse? Nature 328, 123-126 (1987),

16. Maier-Reimer, E., Mikolajewicz, U. \& Winguth, A. Future ocean uptake of $\mathrm{CO}_{2}$ : interaction between ocean circulation and biology. Clim. Dyn. 12, 711-721 (1996).

17. Wright, D. G. \& Stocker, T. F. Sensitivities of a zonally averaged global ocean circulation model. J. Geophys. Res. 97, 12707-12730 (1992).

18. Stocker, T. F., Wright, D. G. \& Mysak, L. A. A zonally averaged, coupled ocean-atmosphere model for paleoclimate studies. J. Clim. 5, 773-797 (1992).

19. Wright, D. G., Vreugdenhil, C. B. \& Hughes, T. M. Vorticity dynamics and zonally averaged ocean circulation models. J. Phys. Oceanogr. 25, 2141-2154 (1995).

20. Chen, D., Gerdes, R. \& Lohmann, G. A 1-D atmospheric energy balance model developed for ocean modelling. Theor. Appl. Climatol. 51, 25-38 (1995).

21. Weaver, A. J., Marotzke, J., Cummins, P. F. \& Sarachik, E. S. Stability and variability of the thermohaline circulation. J. Phys. Oceanogr. 23, 39-60 (1993).

22. Schiller, A., Mikolajewicz, U. \& Voss, R. The stability of the thermohaline circulation in a coupled ocean-atmosphere general circulation model. Clim. Dyn. 13, 325-347 (1997).

23. Egger, J. Flux correction: tests with a simple ocean-atmosphere model. Clim. Dyn. 13, 285-292 (1997).

24. Shine, K. P., Derwent, R. G., Wuebbles, D. J. \& Morcrette, J.-J. in Climate Change: The IPCC Scientific Assessment (eds Houghton, J. Y., Jenkins, G. J. \& Ephraums, J. J.) 41-68 (Cambridge Univ. Press, 1990)

25. Johns, T. C. et al. The second Hadley Centre coupled ocean-atmosphere GCM: model description, spinup and validation. Clim. Dyn. 13, 103-134 (1997)

26. Mikolajewicz, U. \& Maier-Reimer, E. Mixed boundary conditions in ocean general circulation models and their influence on the stability of the model's conveyor belt. J. Geophys. Res. 99, 22633-22644 (1994).

27. Rahmstorf, S. Bifurcations of the Atlantic thermohaline circulation in response to changes in the hydrological cycle. Nature 378, 145-149 (1995).

28. Rahmstorf, S., Marotzke, J. \& Willebrand, J. in The Warmwatersphere of the North Atlantic Ocean (ed. Krauss, W.) 129-157 (Bornträger, Berlin, 1996)

29. Wigley, T. M. L., Richels, R. \& Edmonds, J. A. Economic and environmental choices in the stabilization of atmospheric $\mathrm{CO}_{2}$ concentrations. Nature 379, 240-243 (1996).

Acknowledgements. We thank N. Gruber, F. Joos, O. Marchal, H. Oeschger, S. Rahmstorf, A. Weaver and D. Wright for comments. This work was supported by the Swiss National Science Foundation.

Correspondence should be addressed to T.F.S. (e-mail: stocker@climate.unibe.ch).

\section{Critical behaviour and the evolution of fault strength during earthquake cycles}

\section{Moritz Heimpel}

Institut für Geophysik, Universität Göttingen, D-37075 Göttingen, Germany

The problem of how fault rheology and heterogeneity interact to produce the observed scaling of earthquakes (such as the powerlaw moment-frequency relationship) remains largely unsolved. Rock friction experiments have elucidated the properties of smooth faults ${ }^{1-3}$, but seem insufficient to explain the observed complexity of real fault dynamics ${ }^{4,5}$. The recognition of a connection between fault-related processes and critical phenomena in other physical systems, together with numerical models of repeated earthquakes, have resulted in significant progress in the theoretical interpretation of earthquake scaling ${ }^{4-14}$. But fault rheology and heterogeneity have so far been treated separately. Here I attempt to unify the requirements of fault rheology and heterogeneity using numerical calculations of quantized slip in an elastic continuum. I show that cyclical fault strength evolves naturally by means of a statistical selection for high-strength fault patches (asperities), resulting in the accumulation and eventual failure of those asperities. The applicability of these results to real fault systems is supported by a recent analysis of time-dependent earthquake statistics ${ }^{15}$. These results imply that self-similarity and criticality on a fault emerge during an earthquake cycle, and suggest that the character of local seismicity can be useful in earthquake forecasting by revealing how advanced a fault is within its cycle.

During an earthquake the state of the fault-crust system changes rapidly. Stress is released and the fault strength drops. Between earthquakes, fault stress and strength recover through slip- and time-dependent healing. The physics of this cyclical process can be understood in terms of the evolution of a population of isolated contact surfaces $^{16-18}$. Such a population arises when two rough fault surfaces are pressed together with insufficient normal force to close apertures. An important parameter controlling this evolution is the minimum critical slip $L_{\min }$ required to change the strength of a population of contacts. Although fault surfaces are fractal and selfaffine ${ }^{19-21}$, it has been shown that two unmatched surfaces pressed together produce a maximum distance $\lambda_{c}$ between contact surfaces (ref. 16), implying a minimum critical slip distance $L_{\min } \approx \lambda_{c}$. Estimates of $\lambda_{c}$ (and thus $L_{\text {min }}$ ) for natural fault surfaces typically range from $10^{-3}$ to $10^{-2} \mathrm{~m}$ (refs $16-18$ ). For natural faults the presence of gouge also affects the scaling of $L_{\text {min }}$; although not explicitly treated here, its effect is analogous to increasing fault surface roughness ${ }^{22}$.

In the numerical model presented here, $\lambda_{c}$ (and thus also $L_{\min }$ ) is further interpreted to represent the minimum slip required to statistically decorrelate the local yield strength on a fault patch. This is clearly applicable if fault heterogeneity results from a diversity of loading and geometrical conditions on the scale of individual contacts. The model uses exact solutions for quantized slip on a fault surface embedded in a three-dimensional elastic medium. The model may be classified as inherently discrete (as defined in ref. 4) because, as discussed below, the yield strength of individual model cells can change abruptly. The main assumptions are as follows. (1) Inertia is neglected (quasi-static approximation). (2) Background stress increases uniformly over the fault plane. Time steps are chosen so that a quake initiates at a single numerical cell at every time step. (3) Slip is quantized; each of the $\sim 10^{8}$ discrete slips 
in a simulation contributes an identical slip increment $L$ and stress field $\sigma_{\mathrm{L}}$ to the fault domain. (4) Boundary conditions are periodic.

The model fault consists of $200 \times 200$ square cells. The shear stress at a cell centre is

$$
\tau_{k}=\sum_{l}\left(\sigma_{k}\right)_{l} n_{l}+\sigma_{0}(t)
$$

where $\left(\sigma_{k}\right)_{l}$ is the shear stress at cell location $k$ due to the displacement discontinuity (that is, slip) $\Delta u$ at cell $l, n_{l}$ is the number of slip increments at cell $l$, and $\sigma_{0}(t)$ is the uniformly increasing shear stress. Periodic boundary conditions are imposed in the following way (see also ref. 4). The stress field due to each slip increment is

$$
\begin{aligned}
\sigma\left(i-i^{\prime}, j-j^{\prime}\right)= & \sum_{p=-m}^{+m} \sum_{q=-m}^{+m} \sigma^{\prime}\left(i-i^{\prime}-p, j-j^{\prime}-q\right) \\
& -\phi \delta\left(i-i^{\prime}-p, j-j^{\prime}-q\right)
\end{aligned}
$$

where $\sigma^{\prime}\left(i-i^{\prime}, j-j^{\prime}\right)$ is the shear stress at location $(i, j)$ in the slip plane due to a single slip increment located at $\left(i^{\prime}, j^{\prime}\right)$ in a threedimensional full space, $p=q$ is the number of cells defining the width of the square numerical domain, $\phi$ is a dissipation parameter, and $\delta$ is the Kronecker delta function. For an infinite, periodically repeating stress field, $m=\infty$. Here, $m=30$ is used as a good approximation.

There are several mechanisms by which faults dissipate energy, including viscous heating, distributed fracture and seismic radiation. However, to keep the interpretation of dissipation simple in the context of the quasi-static approximation, $\phi$ is set to release the amount of spatially averaged stress that would be lost out of a similar model fault patch with open boundaries, due to elastic stress transfer out of the model region. Thus

$$
\phi=\left\langle\sum_{i=-\infty}^{\infty} \sum_{j=-\infty}^{\infty} \sigma^{\prime}\left(i-i^{\prime}, j-j^{\prime}\right)-\sum_{i=-p / 2}^{p / 2} \sum_{j=-q / 2}^{q / 2} \sigma^{\prime}\left(i-i^{\prime}, j-j^{\prime}\right)\right\rangle_{(3)}
$$

where the angle brackets indicate that the expression on the righthand side of equation (3) is averaged over the model fault area. The boundary condition was chosen to best represent a sub-area on a fault of larger extent within the obvious limitations of computer modelling. The main disadvantage of periodic boundary conditions

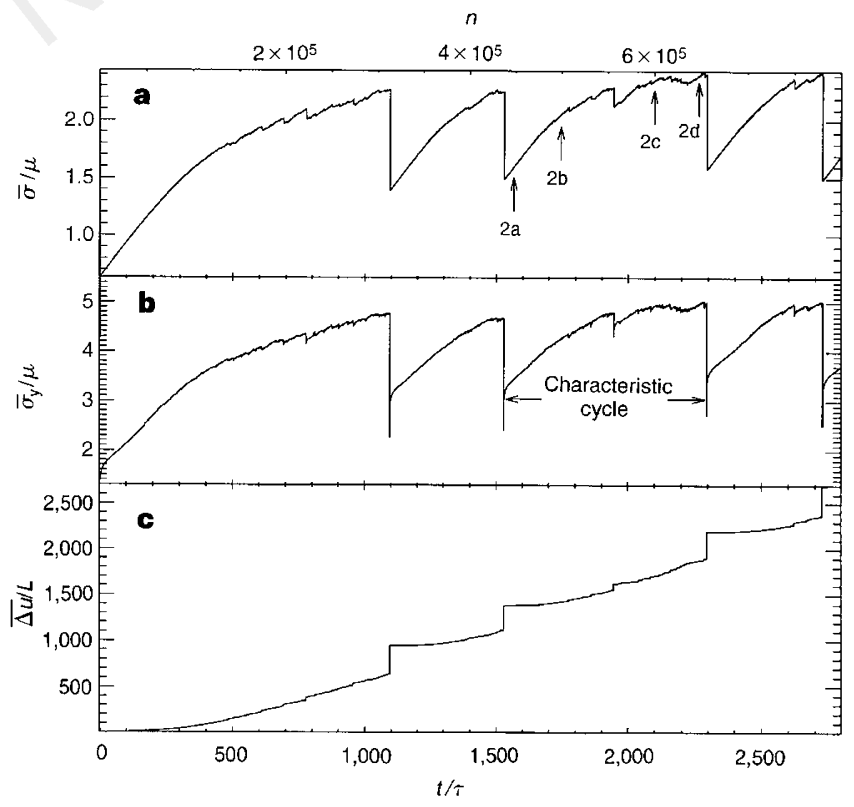

is that wavelengths of deformation longer than the size of the numerical grid are neglected. An advantage is that, unlike the case of open boundary conditions, the stress drop for a single slip increment is independent of its position. As a result there is no time-averaged spatial bias for slip accumulation.

Fault heterogeneity is modelled using a yield-strength probability distribution

$$
p\left(\sigma_{y}\right)=\frac{2 \sigma_{y}}{\lambda^{2}}\left(1+\frac{\sigma_{y}^{2}}{\lambda^{2}}\right)^{-2}
$$

where $\sigma_{\mathrm{y}}$ is the yield strength and $\lambda$ is a scale parameter such that $\mu=(\pi / 2) \lambda$ is the mean. Equation (4) can be derived as a mixture of Weibull distributions and has been shown to be an appropriate model for fault heterogeneity ${ }^{23}$. Note that $\sigma_{y}$ may be interpreted either as the local yield strength or as a yield-strength fluctuation. This can be seen by writing $\sigma_{y}=\delta_{0}+\sigma_{y}^{\prime}$ where $\sigma_{0}$ is constant and $\sigma_{y}^{\prime}$ is the random variable. The choice of $\sigma_{0}$ simply sets the minimum strength and has no other effect on the results. In the numerical procedure $\lambda$ (and hence $\mu$ ) is constant and a standard algorithm using a random-number generator is used to output values of $\sigma_{\mathrm{y}}$ that are distributed according to equation (4). A model quake occurs when the strength of a cell obtains the yield strength, resulting in slip at that cell. Because slip changes the stress field on the numerical fault, one such slip can precipitate a large quake as a cascade of many (up to $\sim 10^{7}$ ) individual slips. For every quake each numerical cell that slips at least once is given a new strength from equation (4) after the initial slip. The new strength value is retained for subsequent slip during that quake. This ad hoc rule is meant to represent brittle failure of asperities, where the strength randomness represents the local heterogeneity associated with freshly fractured surfaces sliding past one another. Because the mean value $\mu$ of newly assigned strengths remains constant throughout the simulation, the probability of strengthening or weakening is proportional to the strength before failure.

The time evolution of domain-averaged fault properties is shown in Fig. 1. Here, a characteristic cycle is defined as the interval between events that rupture nearly the entire numerical domain. Although initial cell strengths are statistically uncorrelated in space and time, spatiotemporal correlations in model fault properties arise naturally from the interaction between the heterogeneity and the mechanics of the model system. Strong clusters of cells remain inactive longer before slipping; thus stronger asperities are selected

Figure 1 Domain-averaged time series of stress $\bar{\sigma} / \mu(\mathbf{a})$, yield strength $\bar{\sigma}_{\mathrm{y}} / \mu(\mathbf{b})$ and slip $\overline{\Delta u} / L$, where $\overline{\Delta u}$ is the domain-averaged slip (c). A uniformly increasing background stress is imposed. The bottom horizontal axis is the dimensionless time $t / \tau$, where $\tau$ is the time required to recover the stress drop due to a single slip increment over the entire fault domain. The top horizontal axis shows the step number $n$ where each step corresponds to a model quake. Initially, yield strength is assigned by the distribution of equation (3), and stress is set at the mean strength $\mu$. This implies that $\bar{\sigma} / \mu=1$ and $\bar{\sigma}_{\mathrm{y}} / \mu=1$ at $t=0$, where domain averages are indicated by an overbar. In the first time step, the average stress falls as all numerical cells with strengths $\sigma_{\mathrm{y}}<\mu$ fail. The arrows marked by $2 \mathrm{a}, 2 \mathrm{~b}, 2 \mathrm{c}$ and $2 \mathrm{~d}$ indicate the times that correspond to the four slip images in Fig. 2. The calculation resulted in $n=8 \times 10^{5}$ quakes with quake sizes ranging from $m=1$ to $m \approx 10^{7}$ where $m$ is the number of quantized slips per quake. Four full characteristic quake cycles occurred during this calculation. After an initial very large event at $n \approx 3 \times 10^{5}$, an asymptotic state obtains where increasing stress and strength are reset cyclically by three additional characteristic events that rupture almost the entire domain. A characteristic cycle begins with a very large quake, which precipitates a stress and strength drop, followed by relatively smooth stress recovery and strength increase (that is, healing) until a critical state is reached. Late in the cycle, large fluctuations in stress and strength correspond to larger model quakes that initiate local quake cycles on increasingly larger spatial scales, culminating in a very large event that initiates another characteristic cycle. 
for and survive longer. As a result, during a quake cycle, a cell population evolves into one with an average yield strength that is significantly higher than the initial mean strength; the domainaveraged strength rises to a maximum of $\bar{\sigma}_{y}=5 \mu$.

The selection process during a characteristic cycle is shown in Fig. 2. Smooth, high-slip regions indicate low yield strength, whereas low-slip areas represent high-strength cell-clusters (asperities). The relative area occupied by asperities increases with time within the cycle.

Slip resulting from a moderately large quake sequence is shown in Fig. 3, illustrating several of the evolutionary phenomena described above. Figures $3 \mathrm{a}$ and $\mathrm{b}$ show quakes occurring at the latest stage of a cycle. The fore-shock (Fig. 3a) to main-shock (Fig. 3b) sequence shows localized slip on an increasing spatial scale that is representative of the model fault late in a characteristic cycle. The after-slip event (Fig. 3c) and the cumulative slip (Fig. 3d) show the transition to the early stage of a new cycle on the freshly slipped patch.

Figure 4 shows the time series of the domain-averaged stressstrength ratio $\bar{\sigma} / \bar{\sigma}_{y}$, clearly illustrating the stages of a model quake
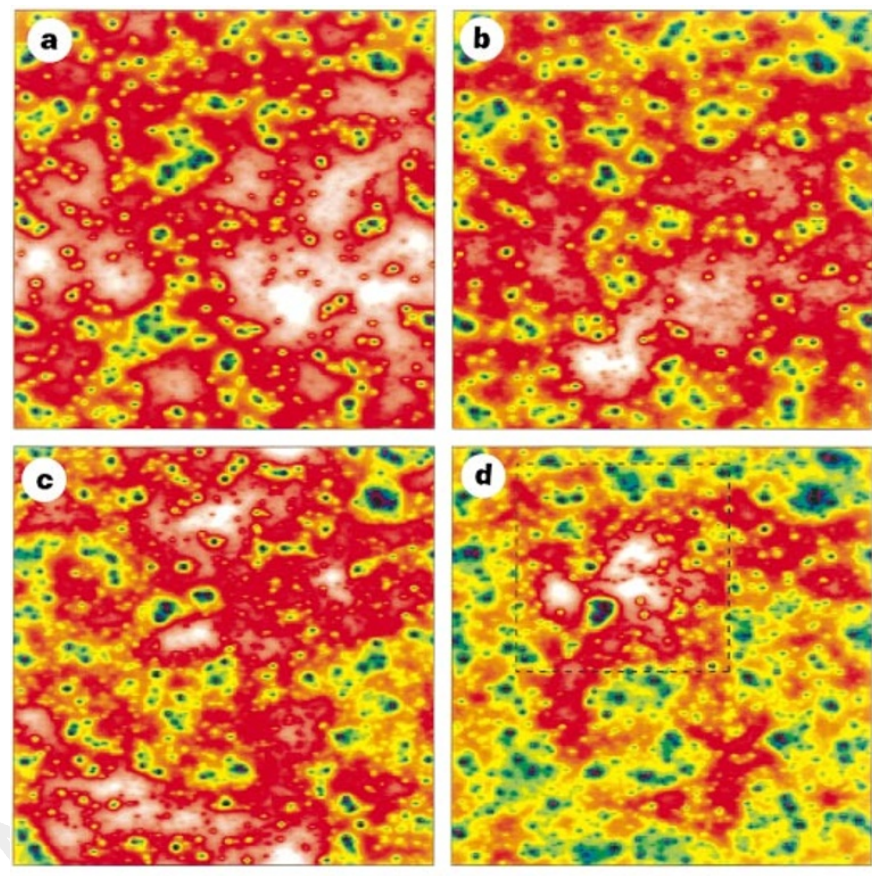

$\operatorname{Max}-500$

$\operatorname{Max}$

$\Delta U / L$

Figure 2 a-d, Images of relative amounts of total slip $\Delta U$ for the four moments in time marked in Fig. 1. Here, $\Delta U=\Delta u+\epsilon \Delta x$, where $\Delta u$ is the slip discontinuity across the fault, $\epsilon$ is the shear strain, and $\Delta x$ is the cell width. Each image shows the entire numerical domain and consists of $200 \times 200$ model cells. The colours for each image scale from the maximum $\Delta U$ (white) to $500 \mathrm{~L}$ less than the maximum (black) for that image. The dashed square in $\mathbf{d}$ indicates the fault area shown in Fig. 3. a, The slip directly after a large event (that is, early in a characteristic cycle). The bright, relatively high-slip regions are 'smooth' as a result of extinction of asperities during the large event. Low-slip areas are indicative of high yield strength and clusters of low-slip cells may be referred to as 'asperities'. Each time a quake occurs, any numerical cell that slips is given a new and uncorrelated strength according to equation (4) (see explanation in text). During a cycle, slip preferentially occurs on weak fault cells so that strong cells and cell clusters have a greater mean lifetime. In this way asperities are selected for so that, late in a cycle, much of the model fault surface is composed of asperities (d). When asperities fail, the stress on the bounding fault surface increases in proportion to the integrated strength of the failed asperity. As a result of this proportionality, and the selection process, increasingly large fault patches fail during the course of a quake cycle. cycle. To interpret the difference in size-frequency statistics between early (Fig. 4b) and later (Fig. 4c) stages, note that selfsimilar scaling in the rupture process is implied by a negative slope of $B=2 / 3$ in the moment-frequency relation ${ }^{24,25}$. Early in a quake cycle $B \approx 4 / 3$, indicating lack of self-similarity. Later in the cycle the fault reaches a critical stage where stress and strength fluctuations increase and $B$ approaches $2 / 3$. These results support recent observations of time-variable microseismicity statistics at Parkfield, California, where the latest in an almost periodic (recurrence interval $\sim 22$ years) series of similar-sized large earthquakes (magnitude $\sim 6$ ) is overdue ${ }^{15}$. At Parkfield the $B$-value of microseismicity has decreased from 0.68 to 0.51 over about 4 years.

To summarize the model results, a characteristic quake cycle may be broken up into four stages. (1) Opportunism. The cycle begins with a flurry of relatively small aftershocks following the mainshock sequence of the previous cycle. The average yield strength increases rapidly with a decreasing slope as a fresh population of relatively weak asperities fail and are replaced by stronger ones. (2) Self organization. As the selection of relatively strong fault patches
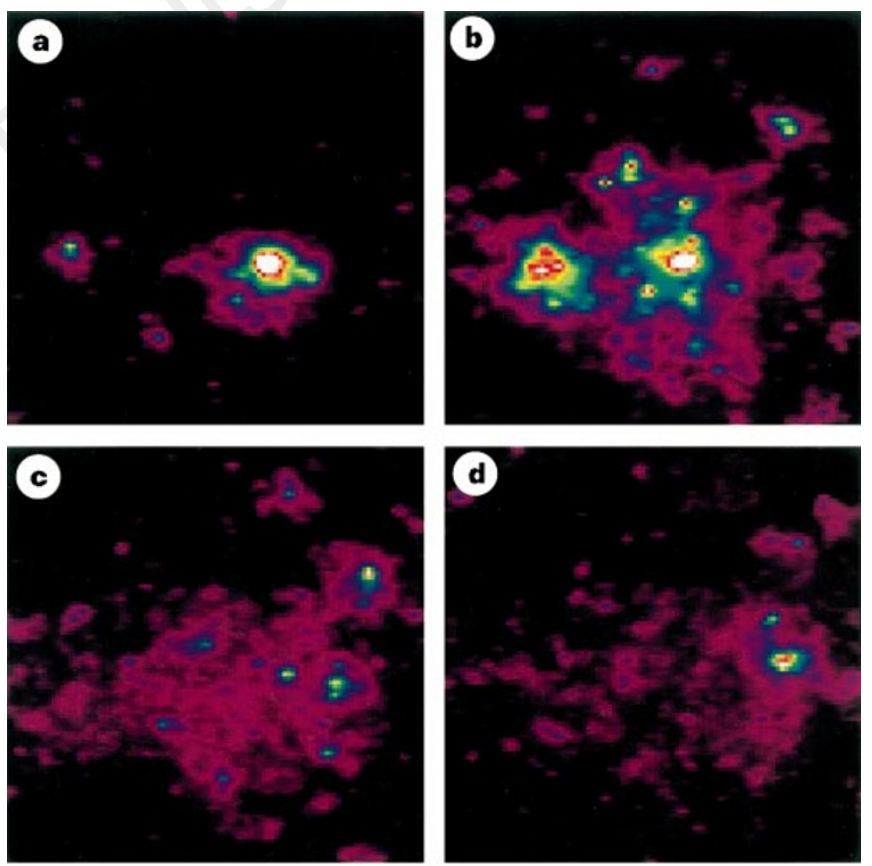

0

$\Delta U / L$

Figure $\mathbf{3} \mathbf{a}-\mathbf{d}$ Images of the total slip $\Delta U$ for a model quake sequence. The area for each image is indicated by the dashed square in Fig. $2 \mathrm{~d}$ and consists of $100 \times 100$ model cells. a, A large model 'fore-shock', characterized by highly localized slip. This typically results when a cluster of high-yield-strength numerical cells (that is, an asperity) fails. $\mathbf{b}$. The slip for the main shock. This event is precipitated by high stresses on the boundary of the fore-shock slip area. c, After-slip occurring on the main shock area, immediately following the main shock. This occurs because the main shock results in the replacement of asperities with cells of a mean strength distribution (given by equation (4)), so that the mean strength on that area is relatively low, and the strength distribution is relatively smooth compared to the surrounding fault area. As this area accumulates further slip, it will strengthen and roughen as asperities are selected for. d, Cumulative slip for 10 smaller 'aftershocks'. Model seismicity is shown to migrate away from the main shock area. 

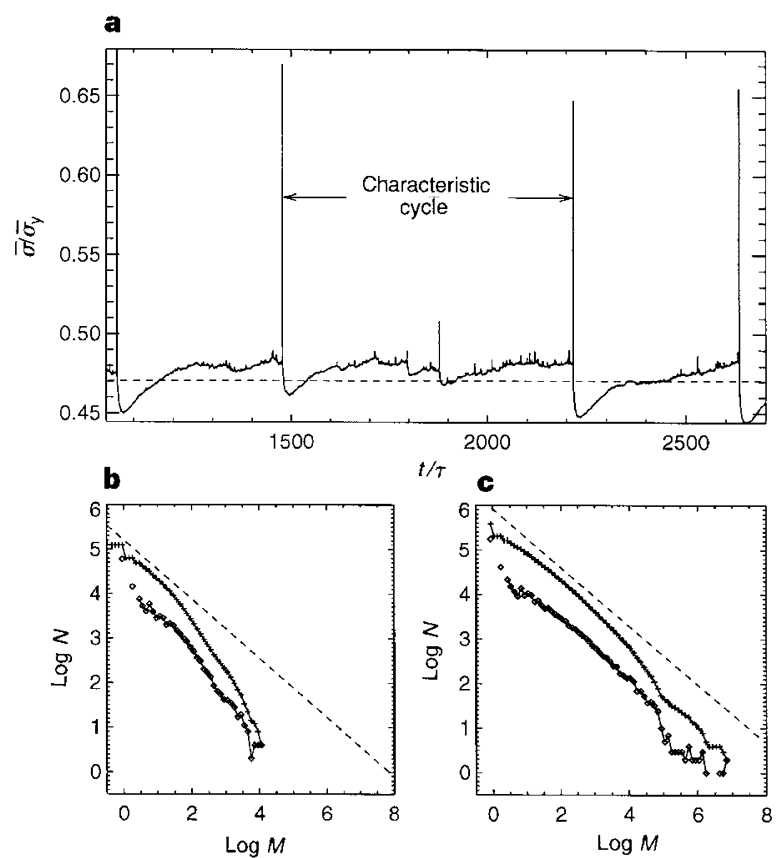

Figure 4 a, Domain-averaged ratio of stress to yield strength $\bar{\sigma} / \bar{\sigma}_{y}$ for $n=(3-8) \times 10^{5}$ for the last three characteristic cycles illustrated in Fig. 1 . This plot clearly shows the stages within model quake cycles. Large events are indicated by sharp peaks in $\bar{\sigma} / \bar{\sigma}_{\mathrm{y}}$. Also shown are the size-frequency statistics for the early stages of quake cycles $\left(\bar{\sigma} / \bar{\sigma}_{y}<0.47 ; \mathbf{b}\right)$, and the later stages $\left(\bar{\sigma} / \bar{\sigma}_{y}>0.47 ; \mathbf{c}\right)$. Immediately following large events, $\bar{\sigma} / \bar{\sigma}_{\mathrm{y}}$ decreases rapidly as the weakest cells from a new population fail. The stress and strength continue to self-organize as fluctuations increase; the emergence of a critical stage is marked by a levelling-off or decrease of $\bar{\sigma} / \bar{\sigma}_{y}$ at a value above $\sim 0.47$ (indicated by the dashed line). Qualitatively, this occurs when high-strength asperities begin to fail. b, c, Plots of quake size (measured as $\log M$, where $M$ is the absolute number of discrete slips per quake) versus the cumulative number of slips $N$ (crosses) and $N$ binned for every $1 / 10 \log M$ unit (diamonds). The size-frequency statistics for the subcritical state (defined as $\left.\bar{\sigma} / \bar{\sigma}_{\mathrm{y}}<0.47\right)$ and the critical state $\left(\bar{\sigma} / \bar{\sigma}_{\mathrm{y}}>0.47\right)$ are shown in $\mathbf{b}$ and $\mathbf{c}$ respectively. The dashed lines in $\mathbf{b}$ and $\mathbf{c}$ show the negative slope $B=2 / 3$ (corresponding to a Gutenberg-Richter 'b-value' equal to one). Comparing the slopes, it is apparent the Gutenberg-Richter scaling is more closely approximated during the critical than the subcritical state. This shows that self-similarity is violated in the early stages of a quake cycle and emerges during the transition to the critical state.

(asperities) continues, stress and strength increase. (3) Criticality. Here, large stress/strength fluctuations occur as stronger asperities fail, initiating four-stage quake cycles on increasing spatial and temporal scales within the characteristic cycle. (4) Extinction. The characteristic cycle ends with failure of major asperities and a mainshock sequence that spans nearly the entire domain. This is accompanied by a rapid stress and strength drop as the yieldstrength distribution is reinitiated with the mean population strength.

Received 15 January; accepted 21 July 1997.

. Dieterich, J. H. Modeling of rock friction 1. Experimental results and constitutive equations. J. Geophys. Res. 84, 2161-2168 (1979).

2. Ruina, A. Slip instability and state variable friction laws. J. Geophys. Res. 89, 10359-10370 (1983).

3. Beeler, N. M., Tullis, T. E. \& Weeks, J. D. The roles of time and displacement in the evolution effect in rock friction. Geophys. Res. Lett. 21, 1987-1990 (1994).

4. Rice, J. R. Spatio-temporal complexity of slip on a fault. J. Geophys. Res. 98, 9885-9907 (1993).

5. Rice, J. R. \& Ben-Zion, Y. Slip complexity in earthquake fault models. Proc. Natl Acad. Sci. USA $\mathbf{9 3}$ 3811-3818 (1996).

6. Bak, P. \& Tang, C. Earthquakes as a self-organized critical phenomenon. J. Geophys. Res. 94, $15635-$ 15637 (1989).

7. Sornette, D. in Spontaneous Formation of Space-Time Structures and Criticality (eds Riste, T. \& Sherrington, D.) 57-106 (Proc. NATO, ASI, Ser C, 349 Kluwer, Dordrecht, 1991).
8. Xu, H. J., Bergersen, B. \& Chen, K. Self-organized ruptures in an elastic medium: a possible model for earthquakes. J. Phys. A 25, L1251-L1256 (1992).

9. Rundle, J. B. \& Klein, W. Scaling and critical phenomena in a cellular automaton slider-block for earthquakes. J. Statist. Phys. 72, 405-413 (1993).

10. Rundle, J. B., Klein, W. \& Gross, S. Dynamics of a traveling density wave model for earthquakes. Phys. Rev. Lett. 76, 4285-4289 (1996).

11. Main, I. Statistical physics, seismogenesis, and seismic hazard. Rev. Geophys. 34, 433-462 (1996).

12. Burridge, R. \& Knopoff, L. Model and theoretical seismicity. Bull. Seismol. Soc. Am. 57, 341-371 (1967).

13. Rydelek, P. A. \& Sacks, S. Earthquake slip rise time and rupture propagation: Numerical results of the quantum earthquake model. Bull. Seismol. Soc. Am. 86, 567-574 (1996).

4. Ben-Zion, Y. Stress, slip, and earthquakes in models of complex single-fault systems incorporating brittle and creep deformations. J. Geophys. Res. 101, 5677-5706 (1996).

15. Nadeau, R. M. Characterization and application of microearthquake clusters to problems of scaling, fault zone dynamics, and seismic monitoring at Parkfield, California. Thesis, Univ. California, Berkeley, (1995).

16. Scholz, C. H. The critical slip distance for seismic faulting. Nature 36, 761-763 (1988).

17. Scholz, C. H. The Mechanics of Earthquakes and Faulting (Cambridge Univ. Press, New York, 1990). 18. Power, W. L., Tullis, T. E. \& Weeks, J. D. Roughness and wear during brittle faulting. J. Geophys. Res. 93, $15268-15287$ (1988).

19. Power, W. L. \& Tullis, T. E. Euclidean and fractal models for the description of rock surface roughness. J. Geophys. Res. 96, 415-424 (1991).

20. Schmittbuhl, J., Gentier, S. \& Roux, S. Field measurements of the roughness of fault surfaces. Geophys. Res. Lett. 20, 639-641 (1993).

21. Brown, S. R. \& Scholz, C. H. Closure of rock joints. J. Geophys. Res. 91, 4939-4948 (1985).

22. Marone, C. \& Kilgore, B. Scaling of the critical slip distance for seismic faulting with shear strain in fault zones. Nature 362, 618-621 (1993).

23. Heimpel, M. Earthquake size-frequency relations from an analytical stochastic rupture model. $J$. Geophys. Res. 101, 22435-22448 (1996).

24. Andrews, D. J. A stochastic fault model 1. Static case. J. Geophys. Res. 85, 3876-3877 (1980).

25. Rundle, J. Derivation of the complete Gutenberg-Richter magnitude-frequency relation using the principle of scale invariance. J. Geophys. Res. 94, 12337-12342 (1989).

Acknowledgements. I thank M. M. Wheelock for discussions. This work was supported in part by the Deutsche Forschungsgemeinschaft. Additional research was carried out during a visit to Johns Hopkins University, supported by the National Science Foundation.

Correspondence should be addressed to the author (e-mail: moritz@willi.uni-geophys.gwdg.de).

\section{The Late Precambrian fossil Kimberella is a mollusc-like bilaterian organism}

\section{Mikhail A. Fedonkin $*$ \& Benjamin M. Waggoner $\dagger$}

* Paleontological Institute, Russian Academy of Sciences, ul. Profsoyuznaya 123, Moscow 117647, Russia

$\dagger$ Department of Integrative Biology, University of California, Berkeley, California 94720, USA

The fossil Kimberella quadrata was originally described from late Precambrian rocks of southern Australia'. Reconstructed as a jellyfish ${ }^{2}$, it was later assigned to the cubozoans ('box jellies'), and has been cited as a clear instance of an extant animal lineage present before the Cambrian ${ }^{3-7}$. Until recently, Kimberella was known only from Australia, with the exception of some questionable north Indian specimens ${ }^{8}$. We now have over thirty-five specimens of this fossil from the Winter Coast of the White Sea in northern Russia. Our study of the new material does not support a cnidarian affinity. We reconstruct Kimberella as a bilaterally symmetrical, benthic animal with a non-mineralized, univalved shell, resembling a mollusc in many respects. This is important evidence for the existence of large triploblastic metazoans in the Precambrian and indicates that the origin of the higher groups of protostomes lies well back in the Precambrian.

The Winter Coast outcrops (Ust'-Pinega Formation) contain a rich 'Ediacara-type' biota ${ }^{9,10}$. Kimberella occurs in several facies all through the section, but most specimens have come from the undersides of fine-grained sandstone gutter casts, in member 9 of the local stratigraphic section ${ }^{10}$. Channels in a clay substrate were colonized by a rich biota; when the channels were rapidly infilled with sand, the biota was preserved in situ ${ }^{11}$. Associated with Kimberella in member 9 are large round 'medusoids', the distinctive fossils Tribrachidium and Dickinsonia, and some undescribed forms. 